\title{
Predicting Local Transport Coefficients at Solid-Gas Interfaces
}

\author{
Nils E. R. Zimmermann, ${ }^{*}{ }^{\dagger}$ Berend Smit, ${ }^{\ddagger}$ and Frerich J. Keil ${ }^{\dagger}$ \\ ${ }^{\dagger}$ Institute for Chemical Reaction Engineering, Hamburg University of Technology, Eissendorfer Str. 38, 21073 Hamburg, Germany \\ ${ }^{\ddagger}$ Department of Chemical Engineering and Department of Chemistry, University of California-Berkeley, 101 B Gilman Hall, \\ Berkeley, California 94720-1462, United States
}

\section{Supporting Information}

\begin{abstract}
The regular nanoporous structure make zeolite membranes attractive candidates for separating molecules on the basis of differences in transport rates (diffusion). Since improvements in synthesis have led to membranes as thin as several hundred nanometers by now, the slow transport in the boundary layer separating bulk gas and core of the nanoporous membrane is becoming increasingly important. Therefore, we investigate the predictability of the coefficient quantifying this local process, the surface permeability $\alpha$, by means of a two-scale simulation approach. Methane tracer-release from the one-dimensional

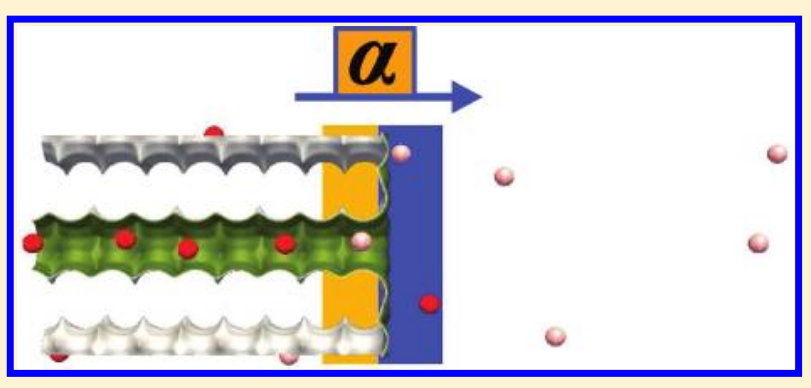
nanopores of an AFI-type zeolite is employed. Besides a pitfall in determining $\alpha$ on the basis of tracer exchange, we, importantly, present an accurate prediction of the surface permeability using readily available information from molecular simulations. Moreover, we show that the prediction is strongly influenced by the degree of detail with which the boundary region is modeled. It turns out that not accounting for the fact that molecules aiming to escape the host structure must indeed overcome two boundary regions yields too large a permeability by a factor of $1.7-3.3$, depending on the temperature. Finally, our results have far-reaching implications for the design of future membrane applications.
\end{abstract}

\section{INTRODUCTION}

Molecular exchange between a gas reservoir and a nanoporous crystalline solid (e.g., a zeolite membrane or crystal) represents a key design process in applications, such as adsorption, molecular-sieving, catalysis, and ion-exchange. Over the last few decades, a good understanding has been developed with regard to the role $\mathrm{e}^{1}$ and dependence ${ }^{2-7}$ of guest diffusion in such regular host structures, that is, the transport mechanism of molecules inside the nanopores far away from the interface to the fluid phase. ${ }^{8,9}$ For example, gas diffusion in zeolites is known to be an activated process ${ }^{4-7}$ where molecules need to overcome a series of regularly distributed internal diffusion barriers which arise from nanopore shape in the unit cell alone. ${ }^{3}$ Many phenomena, including the loading-dependence of the self-diffusion coefficient, can be explained by the variation of such (free) energy barriers. In this context, molecular simulations have been proven to be invaluable, ${ }^{4,5,10}$ owing to improved agreement with experiments. ${ }^{11-13}$ Despite these accomplishments, there are still unresolved problems, ${ }^{14}$ many of which are related to the boundary layer separating the gasphase region from the core zeolite space.

While cases exist in which the boundary layer may accelerate molecular exchange between the reservoir and the porous host, ${ }^{15,16}$ it usually slows down the transport rate close to the surface, ${ }^{6,7,17-20}$ leading to the name of this phenomenon: surface barriers. Exciting insights into their nature have been unraveled only recently. ${ }^{6,7,20}$ Microscopy experiments ${ }^{6}$ in conjunction with mesoscopic modeling ${ }^{7}$ evidenced that exceptionally few accessible pore entrances together with a large number of lattice defects (i.e., vast crystal nonidealities as depicted in Figure 1a) formed extremely high surface barriers

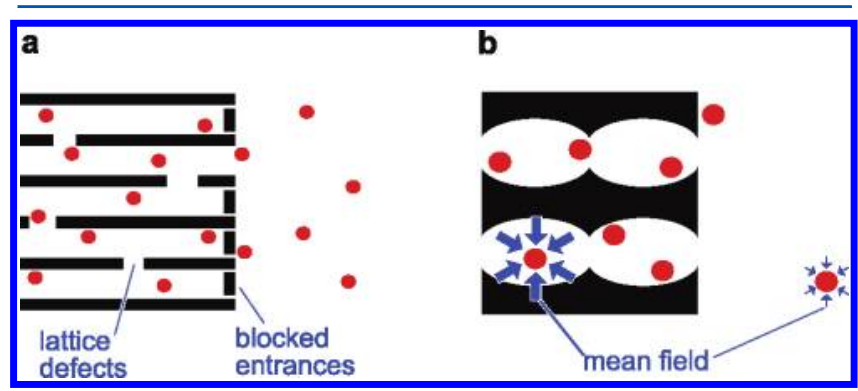

Figure 1. Surface barriers can have different reasons. Blocked pore entrances together with lattice defects (a) and mean-field differences (b) "felt" by molecules in adsorbed and gas space, respectively, can both render the rate of transport at the solid-gas interface extremely small.

on very large MOF $\mathrm{Zn}$ (tbip) crystals (labeled "defect barriers" here). On the other hand, we have recently shown with the aid of molecular simulations ${ }^{20}$ that perfect single-crystal zeolite membranes (i.e., highly ideal crystal and surface structure) can also possess strong barriers at the external surface, as long as thin membranes ( $\lesssim 100$ unit cells) are being considered. The

Received: June 18, 2012

Revised: August 8, 2012

Published: August 10, 2012 
origin of these barriers lay in the difference of the mean field experienced by the guest molecules in gas and adsorbed phase (Figure $1 \mathrm{~b}$ ), for which reason they are labeled intrinsic barriers in the following. Therefore, a unique source of surface barriers does obviously not exist, emphasizing the necessity of a consequent discrimination between different effects.

Despite the improvement of understanding different sources of surface barriers, an assessment of the coefficient quantifying the rate of transport in the boundary layer - the surface permeability $\alpha-$ is so far possible to a satisfactory degree for the defect barriers only. In numerous case studies, Kärger, Chmelik, and co-workers ${ }^{6,17,18,21,22}$ successfully measured the surface permeabilites of gas molecules in large crystals. However, the consistent prediction of this coefficient for intrinsic surface barriers via information from molecular simulations is a challenging task to date. ${ }^{19,23-25}$ This paper therefore aims at highlighting a pitfall that might have been overlooked so far and, most importantly, to provide a satisfying prediction of the surface permeability over those conditions that are currently of practical interest.

\section{METHODOLOGY}

We focus on the conceptually simplest case of molecular exchange, tracer exchange, where macroscopic concentration gradients are absent and the exchange involves differently "colored" molecules rather than different species (Figure 2a and movie in Supporting Information). Since tracer exchange

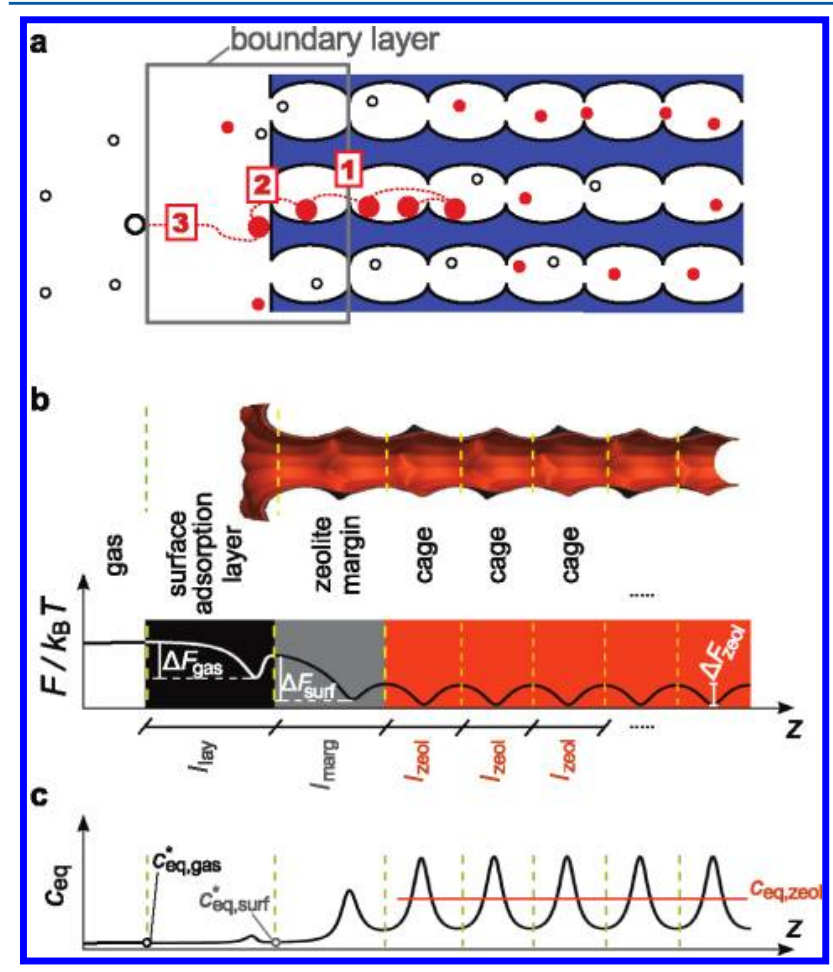

Figure 2. (a) Exchange of a tracer molecule involves three steps: (1) Diffusion to reach the outermost cage (zeolite margin). (2) Jumping out of the pore structure to reach the external surface adsorption layer. (3) Finally, leaving this layer to disappear in the gas phase. (b) Channel and surface structure of the here studied AFI-type zeolite together with a representative free-energy profile, $F / k_{\mathrm{B}} T$, along the exchange direction, $z$. (c) Equilibrium concentration profile, $c_{\text {eq, }}$ corresponding to $(\mathrm{b})$; note that $c_{\mathrm{eq}}(z)=\exp \left[-F(z) / k_{\mathrm{B}} T\right] \times$ const. situations correspond directly to self-diffusion, all diffusion coefficients presented here are self-diffusivities $\left(D_{\mathrm{S}}\right)$.

Several steps are necessary for molecules to be exchanged between zeolite space and gas-phase region, as illustrated by the trajectory of a single molecule in Figure 2a:

1 A molecule needs to traverse the nanopore to eventually reach the outermost cage referred to as the zeolite margin in the remainder. This intracrystalline diffusion is impeded by successive barriers originating from the repetitive crystal structure (internal diffusion barriers, $\Delta F_{\text {zeol }}$, as seen at the end of the orange region of Figure $2 b)$.

2 The molecule must then get out of the pore and reside on the external surface adsorption layer. This process is controlled by a first boundary barrier, $\Delta F_{\text {surf }}$ (gray region in Figure 2b).

3 Finally, to truly desorb from the solid and thus to lose the color, the molecule must overcome a second boundary barrier, $\Delta F_{\text {gas }}$, that separates the surface adsorption layer from the gas-phase region, as evidenced by Figure $2 \mathrm{~b}$ (black region).

Step 1 is mathematically described by the standard Fickian diffusion equations. Steps 2 and 3 are usually modeled with a surface evaporation boundary condition, ${ }^{26}$ yielding a relationship between the current net flux of tracer molecules at the boundary, $j_{\text {surf }}(t)$, the surface permeability, and the driving force:

$$
j_{\text {surf }}(t)=\alpha\left[c_{\text {surf }}(t)-c_{\text {surf }}(t \rightarrow \infty)\right]
$$

where $c$ denotes concentration of tracer (i.e., colored) molecules and $c_{\text {surf }}(t \rightarrow \infty)=0$ in the present case because we consider complete exchange. ${ }^{26}$ At this point, a first problem arises because we obviously need to describe two different processes (steps 2 and 3 ) with a single coefficient $(\alpha)$, which represents one of the main tasks of the present work. However, we consider the release in the first part of the analysis to proceed via a one-step mechanism in which molecules are assumed to be exchanged when they have performed step 2 in Figure 2a. This is instructive because the approximation has been made often in the past but it is not free of problems, as mentioned earlier. Moreover, the resulting one-step surface permeability prediction helps in fact rationalizing the final twostep permeability.

The example of methane desorbing from siliceous AFI-type zeolite membranes will be employed, the structure ${ }^{27}$ of which exhibits one-dimensional channels (Figure 2b). The methodology introduced in ref 20 is extended, as described in detail in the Supporting Information (SI1). It briefly comprises two stages, each involving a different maximum length scale: (i) molecular-detailed simulations where AFI membranes of $\approx 5$ $\mathrm{nm}$ thickness are used and (ii) a continuum calculation to reach membranes of up to several micrometer width. The first stage, where methane is modeled as a united atom, ${ }^{28}$ yields freeenergy and concentration profiles (Figure 2) from which the input data to the second stage are extracted, notably equilibrium transport rates between the different compartments (zeolite cages, margin, surface adsorption layer) as well as their equilibrium concentrations and widths. In the second stage, the material balances are solved numerically on the basis of the data obtained from the molecular simulations. These calculations provide us with transient concentration profiles (Figure 3a). Integral tracer-release curves, $1-m(t) / m(0)$, are then 


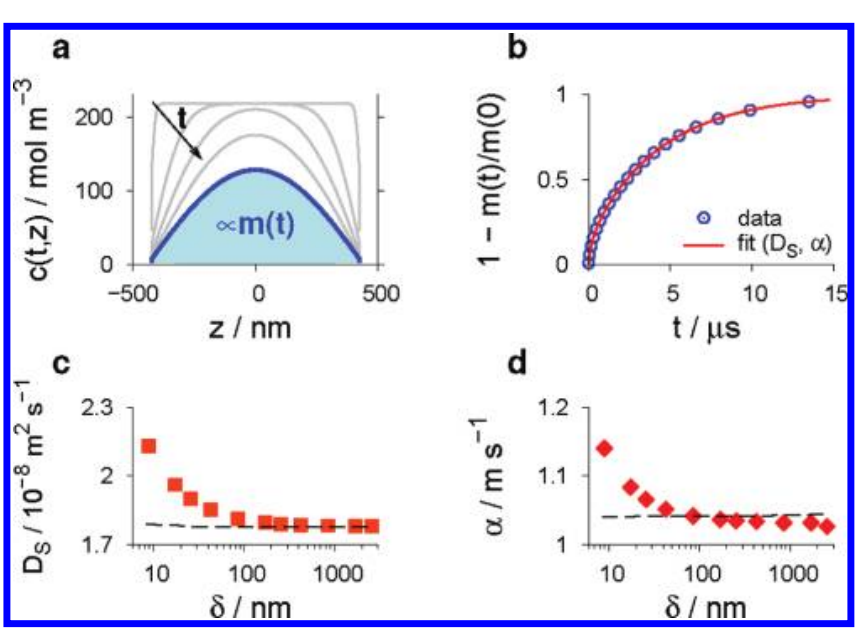

Figure 3. Concentration profiles (a) obtained from a continuum calculation (one-step release mechanism, $\delta=848 \mathrm{~nm}$ ) yield the tracerexchange curve (b). The fitted self-diffusion coefficients (c) and surface permeabilities $(\mathrm{d})$ depend in fact on the membrane thickness, $\delta(T=181 \mathrm{~K}, p=1202 \mathrm{~Pa})$.

determined (Figure 3b), describing the progress of exchanging "colored" molecules (red circles in Figure 2a) for "uncolored" molecules (open circles). Such exchange curves are commonly encountered in diffusion-experiment analysis, ${ }^{29}$ and they can be envisioned in an integral modeling approach to pressure swing adsorption. ${ }^{30}$

The release curves are fitted to the analytical solution ${ }^{26}$ of Fick's laws under consideration of the evaporation boundary condition (eq 1):

$$
1-\frac{m(t)}{m(0)}=1-\sum_{i=1}^{\infty} \frac{2 L^{2} \exp \left[-\gamma_{i}^{2} D_{\mathrm{S}} t /(\delta / 2)^{2}\right]}{\gamma_{i}^{2}\left(\gamma_{i}^{2}+L^{2}+L\right)}
$$

with $L=(\delta / 2) \alpha / D_{S}, \delta$ the membrane thickness, and $\gamma_{i}$ are the positive roots of $\gamma \tan \gamma=L$. The thus obtained transport coefficients are plotted in Figure $3 \mathrm{c}$ and $\mathrm{d}$ for increasing membrane thickness and a single state point $(T, p)$. Note again that these results are obtained from one-step release calculations.

The here presented two-stage simulation strategy features two distinct advantages. First, simulations with membrane thicknesses up to the micrometer range become feasible which would not be possible with conventional equilibrium molecular dynamics (MD), ${ }^{23}$ let alone nonequilibrium $\mathrm{MD}^{31}$ employing thick zeolite membranes. Second, the condition of tracer exchange avoids the possibility that the surface permeability is process-dependent, that is, whether molecule release or uptake is being investigated (cf., Supporting Information SI2 and ref 18.). Therefore, we can focus on the concentration dependence of the permeability which is indeed speculated to be the reason why $\alpha$ varies for uptake and release in nonequilibrium conditions. ${ }^{18}$

\section{RESULTS}

One-Step Release Mechanism. It might seem trivial to match the surface permeability from the continuum calculation with predictions based solely on information from the first stage (molecular simulations). Figure 3 shows however that both $D_{S}$ and $\alpha$ are dependent on the membrane thickness for a given state point. Theoretically, the parameters must not vary with membrane thickness. When we repeat the continuum simulations by setting the zeolite margin width equal to the cage separation $\left(l_{\text {marg }}=l_{\text {zeol }}\right)$ and also equating the equilibrium concentration in the margin with the one of the bulk-zeolite space $\left(c_{\text {eq,marg }}=c_{\text {eq,zeol }}\right)$, the horizontal dashed lines in Figure 3 are obtained, where the diffusivity equals the prediction by dynamically corrected transition state theory. ${ }^{19,32-34}$ With these assumptions, the transport coefficients do not change with thickness, illustrating an important point. The dependence of the transport coefficients on the membrane thickness is brought about by subtle differences of the properties of the zeolite margin from the properties of the inner cages. The maximal deviation of $D_{\mathrm{S}}$ and $\alpha$ amounts to $20 \%$ and $10 \%$, respectively. This underlines the necessity for an improved analytical integral solution of the diffusion process incorporating varying margin width and equilibrium concentration into the boundary condition. The effect, however, can so far not have had any immediate implication to membranes that are currently producible because their thicknesses ${ }^{35}$ are yet more than a magnitude apart from the region where it becomes significant. But it will come into play in the future because improvements in membrane synthesis adumbrate constantly decreasing thicknesses. ${ }^{36,37}$

The practically relevant membrane width range is several hundred nanometers and onward ${ }^{35,37}$ for which both transport coefficients show asymptotic values (Figure $3 c$ and $d$ ). This means that the determination of the surface permeability would involve an entire set of continuum calculations to identify when $\alpha$ becomes constant or, alternatively, a long simulation with a very thick membrane. Instead, one wants to skip this stage and calculate $\alpha$ directly based on data of the first (molecular-level) stage. In this context, we tested three different models. Figure 4 summarizes the results where the line corresponds to (target) surface permeabilities obtained from tracer-exchange continuum calculations with very thick membranes and different symbols represent different models.

The first model (triangles in Figure 4) was suggested by Kärger and co-workers, ${ }^{6,7}$ and assumes that the surface

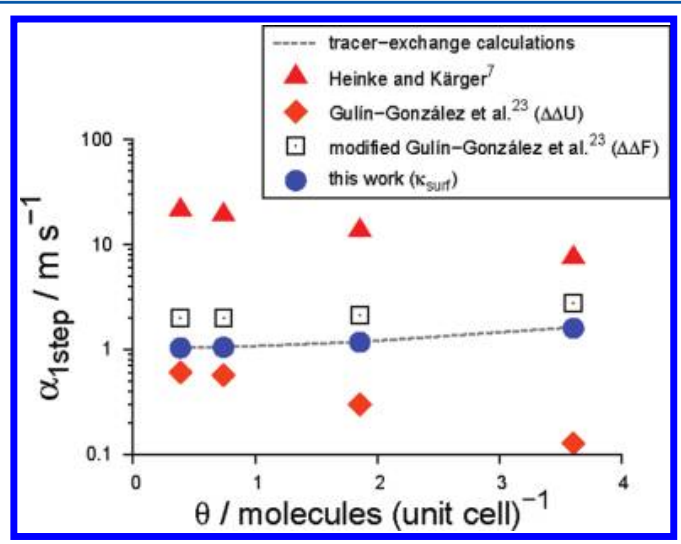

Figure 4. Surface permeability as a function of loading $(T=181 \mathrm{~K})$, obtained from the two-stage simulation approach (line) used in the present work to mimic tracer-exchange experiments. Permeability predictions using information from the first, the molecular, simulation stage only are presented as symbols where different models are tested. Note, first, that the main difference between the modified estimate by Gulín-González et al. ${ }^{23}$ and our prediction lies in $\kappa_{\text {surf }}$ because of the earlier mentioned relationship of free energy and concentration and, second, the molecules are assumed being exchanged when they arrived on the external surface adsorption layer (one-step desorption mechanism). 
permeability is proportional to the diffusion coefficient $(\alpha=$ $\left.0.5 D_{\mathrm{S}} / l_{\text {zeol }}\right)$. While the surface permeability obtained from the tracer-exchange calculations increases with loading (line), the model predicts a declining trend of $\alpha$ because the corresponding diffusion coefficient decreases with loading. Hence, the model of Kärger and co-workers is not applicable for the here studied systems. Since it was validated against diffusion experiments probing defect barriers, the qualitative discrepancy emphasizes again the imperative of discriminating between different surface-barrier sources. As a consequence, different local transport coefficients are obtained just naturally, depending on the relative significance of the two different influences (intrinsic vs. defect barriers).

Gulín-González et al. ${ }^{23}$ suggested that a correlation between the two transport coefficients should be corrected by inclusion of an additional potential-energy barrier. This is because a molecule experiences a higher barrier, $\Delta U_{\text {surf, }}$ when hopping out from the marginal cage onto the external surface in comparison to the internal diffusion barriers, $\Delta U_{\text {zeol }}[\alpha=$ $\exp \left(-\Delta \Delta U / k_{\mathrm{B}} T\right) D_{\mathrm{S}} / l_{\text {zeol, }}$ with $\left.\Delta \Delta U=\Delta U_{\text {surf }}-\Delta U_{\text {zeol }}\right]$. However, this model does not lead to satisfactory qualitative results either (filled diamonds in Figure 4). What changes the trend of the permeability prediction over loading is the consideration of higher free-energy barriers $[\alpha=\exp (-\Delta \Delta F /$ $\left.\left.k_{\mathrm{B}} T\right) D_{\mathrm{S}} / l_{\text {zeol }}\right]$, labeled modified Gulín-González et al. in Figure 4 (squares). Still, a discrepancy is observed that is due mainly to the transmission coefficient, ${ }^{19,20,38,39} \kappa_{\text {surf }}$, the significance of which is discussed shortly. The accurate prediction of $\alpha$ (blue circles in Figure 4) follows

$$
\alpha_{1 \text { step }}=\bar{v} \kappa_{\text {surf }} c_{\text {eq,surf }}^{*} / c_{\text {eq,zeol }}
$$

where $\bar{v}=\left(k_{\mathrm{B}} T / 2 \pi m_{\mathrm{CH}_{4}}\right)^{1 / 2}$ is the average velocity of the methane molecule in a single direction on the basis of kinetic gas theory, $c_{\mathrm{eq}, \text { surf }}^{*}$ is the equilibrium concentration of molecules at the location of the surface barrier and $c_{\text {eq,zeol }}$ the average guest concentration inside the bulk-zeolite structure ( $\propto$ loading). The additional subscript "eq" facilitates discriminability between concentrations obtained from equilibrium $c$-profiles (i.e., timeinvariant, as seen in Figure 2c) and transient concentrations which do change with time (Figure 3a).

Our surface-permeability prediction is in fact the equivalent to the self-diffusion coefficient estimate in the framework of dynamically corrected transition state theory (dcTST). To realize this, consider the dcTST self-diffusivity: ${ }^{32-34}$

$$
D_{\mathrm{S}}^{\mathrm{dcTST}}=\bar{v} \kappa_{\text {zeol }} \frac{\exp \left[-F\left(z_{\text {zeol }}^{*}\right) / k_{\mathrm{B}} T\right]}{\int_{\text {cage }} \exp \left[-F(z) / k_{\mathrm{B}} T\right] \mathrm{d} z} l_{\text {zeol }}^{2}
$$

The equivalency of the terms $\bar{v} \kappa_{i}$ in eqs 3 and 4 is obvious. Recalling that $c_{\text {eq }} \propto \exp \left(-F / k_{\mathrm{B}} T\right)$ leads directly to the correspondence of the integral in the denominator divided by $l_{\text {zeol }}$ and $c_{\text {eq,zeol }}$, except for a factor which, however, is the same one between the two nominators and thus cancels out exactly.

The role of the transmission coefficient is an important finding because, for predicting the permeability, it is usually neglected that not $100 \%$ of the molecules having arrived on top of the barrier between zeolite margin and surface adsorption layer will in fact end up on the external surface. The error introduced by assuming $\kappa_{\text {surf }}=1$ amounts to a factor of $1.7-2.8$ (cf., Supporting Information SI3) which clearly manifests a profound quantitative impact on the final permeability estimate. We furthermore point out that $\kappa_{\text {surf }}$ may not simply be set to $\kappa_{\text {zeol }}$ and that incorporating $\kappa_{\text {surf }}$ into the modified model by Gulín-González et al. still suffers from small deviations from the tracer-exchange data because $\kappa_{\text {zeol }}$ is dragged along in $D_{\mathrm{S}}$. Finally, note also that $\alpha$ is a system property that depends on the combination of guest molecule and host structure as well as on the properties of the surface and its immediate vicinity. ${ }^{18}$

Two-Step Release Mechanism. The investigation of the one-step surface permeability has shed light on the peculiarities encountered when one aims at predicting $\alpha$. Now we return to the case where the second exchange step is considered too, that is, a molecule must also leave the surface adsorption layer for the gas phase to be considered truly exchanged. Again, we determine the "target" $\alpha_{2 \text { step }}$ from tracer-exchange calculations with thick membranes, with the difference that the surface adsorption layer is added in our continuum calculations. As seen from the excellent agreement between the thus obtained data (lines in Figure 5) and direct molecular-simulation

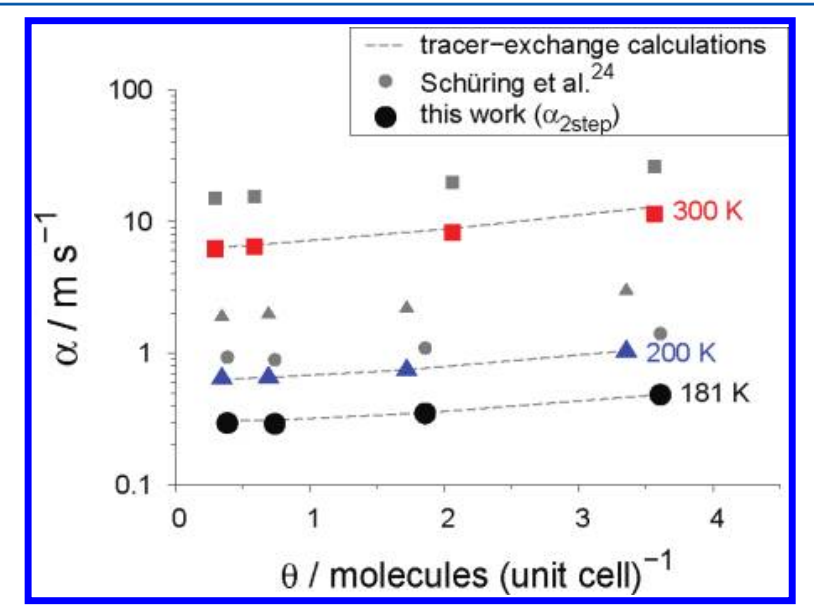

Figure 5. Surface permeability vs loading for the case that tracer release included the desorption from the external surface adsorption layer (two-step release process). Results for various temperatures are shown, where the lines reflect data from the two-stage simulation approach mimicking tracer-exchange experiments and the symbols are predictions on the basis of readily available information from the first, the molecular, simulation stage only. The small gray symbols are surface permeability predictions due to Schüring et al. ${ }^{24}$

predictions (large colored symbols), we find the two-step surface permeability to be given by

$$
\alpha_{2 \text { step }}=\frac{\bar{v} / c_{\text {eq,zeol }}}{1 /\left(\kappa_{\text {gas }} c_{\text {eq,gas }}^{*}\right)+1 /\left(\kappa_{\text {surf }} c_{\text {eq,surf }}^{*}\right)}
$$

where $c_{\text {eq,gas }}^{*}$ is the equilibrium concentration of molecules on the barrier between external surface adsorption layer and gasphase region and $\kappa_{\text {gas }}$ represents its corresponding transmission coefficient; note that $c_{\text {eq,gas }}^{*}=c_{\text {eq,gas }}$ (Figure $2 \mathrm{c}$ ). The total surface permeability hence follows the behavior of parallel resistances in electricity theory, that is, $\alpha_{2 \text { step }}=1 /\left(1 / \alpha_{\text {gas }}+1 /\right.$ $\left.\alpha_{1 \text { step }}\right)$, when one defines $\alpha_{\text {gas }} \equiv \bar{v} \kappa_{\text {gas }} c_{\text {eq,gas }}^{*} / c_{\text {eq,zeol }}$.

The only alternative two-step permeability prediction model that we have found is due to Schüring et al. ${ }^{24}$ (small gray symbols in Figure 5): $\alpha=P_{\text {enter }} \bar{v} / K . K$ denotes the ratio of the average concentrations in the zeolite margin and the bulk gas, $^{24,25}$ and $P_{\text {enter }}$ represents the fraction of molecules that enter the zeolite of all molecules hitting on the surface in total. $^{40}$ While the model captures the qualitative trend of increasing $\alpha$ with loading, it does not predict the permeability 
quantitatively in a satisfactory manner. Deviations from the tracer-exchange calculations range between 2.3 and 3.2, remaining significant even in the case when the transmission coefficients $\left(\kappa_{\text {surf }}\right.$ and $\left.\kappa_{\text {gas }}\right)$ are incorporated into the model $(1.6-2.7)$. At this point, it is important to reiterate that the tracer-exchange surface permeabilities for release and uptake are equivalent (Supporting Information SI2).

\section{DISCUSSION}

A comparison between surface permeabilities obtained from the simplistic one-step and the more realistic two-step mechanism reveals that the external surface adsorption layer substantially retards the desorption from the membrane $\left(\alpha_{2 \text { step }} \ll \alpha_{1 \text { step }}\right)$. The effect becomes the stronger, the lower the temperature gets, and amounts to a factor of $\left(\alpha_{1 \text { step }} / \alpha_{2 \text { step }}\right) 1.7,2.8$, and 3.3 for $T=300,200$, and $181 \mathrm{~K}$, respectively. The comparison clearly underlines that the detailed structure of the membrane boundary layer is exceptionally important to obtain accurate permeability estimates. Since the accuracy is validated against tracer-exchange simulations rather than experiments, this fact deserves some final remarks.

The influence of the here reported intrinsic surface barriers on the permeability vanishes for the giant crystals ${ }^{41}$ employed in microscopy experiments. ${ }^{6}$ This is because the measurements are subject to spatial resolution limitations of around $0.5 \mu \mathrm{m}$ (interference microscopy) and larger (infrared microscopy), and consequently prohibiting the detection of intrinsic surface barriers which level off at this scale. ${ }^{19,20}$ Nevertheless, the question arises to which extent possible defects would play a role, given that those were to occur in ultrathin membranes and thus together with intrinsic barriers. ${ }^{42}$ To provide at least a rough answer to this question, we perform a rule of thumb assessment in the following.

The rating compares the different effects by computing the associated permeabilites and by defining a reference value that corresponds to the limiting case of diffusion-controlled transport $\left(\alpha \propto D_{S}\right)$. Subsequent introduction of impact factors permits a quantitative assessment of the different effects' relative importance because the strength of a certain effect scales directly with the associated surface permeability (Supporting Information SI4 for more details). It turns out that the impact of intrinsic surface barriers varies between 1.5 (almost pure diffusion control observed for high $\theta$ and $T$ ) to 70 (low $\theta$ and $T$ ). An upper bound for the defect-barrier impact was determined to be $\approx 2000$. The simultaneous occurrence of intrinsic barriers and defects leads to a total surface-barrier impact factor of 2000-40000. Clearly, when both effects appear together, the defects exert a stronger influence on the surface barriers than the intrinsic barriers because of the exceptionally large number of closed entrances. However, such a high fraction $(99.95 \%)$ as observed by Hibbe et al. for MOF $\mathrm{Zn}$ (tbip) ${ }^{6}$ is unlikely to occur for the broad AFI pore entrances too (Supporting Information SI4). Furthermore, efficient and effective applications implementating ultrathin membranes (e.g., gas separation, heterogeneous catalysis) usually require high accessibility of guest molecules to the zeolite pores, pointing at improvements of corresponding postsynthesis treatment of the membranes in question.

\section{CONCLUSIONS}

Besides highlighting the need for new integral diffusion solutions, the present work provides an accurate prediction of the local transport coefficient that prevails at solid-gas interfaces: the surface permeability. We have shown that the permeability does not follow the behavior of the associated diffusion coefficient and that it can be very small, especially at low temperature and loading. In this respect, the realization that latest improvements in synthesis enable the production of ultrathin and highly oriented zeolite films ${ }^{37}$ enhances the significance of the here reported slow boundary layer transport. This is because the importance of surface barriers scales inversely with membrane width. ${ }^{20}$ As a final consequence, simple design protocols, such as membrane selectivity formulas on the mere basis of adsorption data and diffusion coefficients, are becoming increasingly inappropriate for the new generation of ultrathin membranes, calling for development of more accurate design models.

\section{ASSOCIATED CONTENT}

\section{S Supporting Information}

Detailed description of two-scale simulation methodology, complementary results, crystal structure, list of symbols; movie illustrating tracer release. This material is available free of charge via the Internet at http://pubs.acs.org.

\section{AUTHOR INFORMATION}

\section{Corresponding Author}

*E-mail: nils.zimmermann@tu-harburg.de.

\section{Notes}

The authors declare no competing financial interest.

\section{ACKNOWLEDGMENTS}

N.E.R.Z. greatly acknowledges the financial support by the German Research Foundation (Deutsche Forschungsgemeinschaft, DFG) in priority program SPP 1155 under contract KE 464/10-1. B.S. was supported as part of the Center for Gas Separations Relevant to Clean Energy Technologies, an Energy Frontier Research Center funded by the U.S. Department of Energy, Office of Science, Office of Basic Energy Sciences under Award Number DE-SC0001015.

\section{REFERENCES}

(1) Hansen, N.; Krishna, R.; van Baten, J. M.; Bell, A. T.; Keil, F. J. J. Phys. Chem. C 2009, 113, 235-246.

(2) Jakobtorweihen, S.; Verbeek, M. G.; Lowe, C. P.; Keil, F. J.; Smit, B. Phys. Rev. Lett. 2005, 95, 044501.

(3) Beerdsen, E.; Dubbeldam, D.; Smit, B. Phys. Rev. Lett. 2006, 96, 044501.

(4) Beerdsen, E.; Dubbeldam, D.; Smit, B. J. Phys. Chem. B 2006, 110, 22754-22772.

(5) Zimmermann, N. E. R.; Jakobtorweihen, S.; Beerdsen, E.; Smit, B.; Keil, F. J. J. Phys. Chem. C 2007, 111, 17370-17381.

(6) Hibbe, F.; Chmelik, C.; Heinke, L.; Pramanik, S.; Li, J.; Ruthven, D. M.; Tzoulaki, D.; Kärger, J. J. Am. Chem. Soc. 2011, 133, 28042807.

(7) Heinke, L.; Kärger, J. Phys. Rev. Lett. 2011, 106, 074501.

(8) Krishna, R. J. Phys. Chem. C 2009, 113, 19756-19781.

(9) Sholl, D. S. Acc. Chem. Res. 2006, 39, 403-411.

(10) Zimmermann, N. E. R.; Haranczyk, M.; Sharma, M.; Liu, B.; Smit, B.; Keil, F. J. Mol. Simul. 2011, 37, 986-989.

(11) Beerdsen, E.; Smit, B. J. Phys. Chem. B 2006, 110, 14529-14530.

(12) Jobic, H.; Theodorou, D. N. J. Phys. Chem. B 2006, 110, 19641967.

(13) Krishna, R. Chem. Soc. Rev. 2012, 41, 3099-3118.

(14) Gueudre, L.; Jolimaite, E.; Bats, N.; Dong, W. Adsorption 2010, $16,17-27$. 
(15) Whitmore, L.; Slater, B.; Catlow, C. R. A. Phys. Chem. Chem. Phys. 2000, 2, 5354-5356.

(16) Gobin, O. C.; Reitmeier, S. J.; Jentys, A.; Lercher, J. A. J. Phys. Chem. C 2011, 115, 1171-1179.

(17) Heinke, L.; Kortunov, P.; Tzoulaki, D.; Kärger, J. Phys. Rev. Lett. 2007, 99, 228301.

(18) Chmelik, C.; Varmla, A.; Heinke, L.; Shah, D. B.; Kärger, J.; Kremer, F.; Wilczok, U.; Schmidt, W. Chem. Mater. 2007, 19, 60126019.

(19) Zimmermann, N. E. R.; Smit, B.; Keil, F. J. J. Phys. Chem. C 2010, 114, 300-310.

(20) Zimmermann, N. E. R.; Balaji, S. P.; Keil, F. J. J. Phys. Chem. C 2012, 116, 3677-3683.

(21) Chmelik, C.; Kärger, J. Chem. Soc. Rev. 2010, 39, 4864-4884.

(22) Tzoulaki, D.; Heinke, L.; Castro, M.; Cubillas, P.; Anderson, M. W.; Zhou, W.; Wright, P. A.; Kärger, J. J. Am. Chem. Soc. 2010, 132, $11665-11670$

(23) Gulín-González, J.; Schüring, A.; Fritzsche, S.; Kärger, J.; Vasenkov, S. Chem. Phys. Lett. 2006, 430, 60-66.

(24) Schüring, A.; Gulín-González, J.; Vasenkov, S.; Fritzsche, S. Microporous Mesoporous Mater. 2009, 125, 107-111.

(25) Combariza, A. F.; Sastre, G. J. Phys. Chem. C 2011, 115, 1375113758.

(26) Crank, J. The Mathematics of Diffusion, 2nd ed.; Oxford University Press: New York, 1975.

(27) Qiu, S.; Pang, W.; Kessler, H.; Guth, J. L. Zeolites 1989, 9, 440444.

(28) Dubbeldam, D.; Calero, S.; Vlugt, T. J. H.; Krishna, R.; Maesen, T. L. M.; Beerdsen, E.; Smit, B. Phys. Rev. Lett. 2004, 93, 088302.

(29) Hibbe, F.; Marthala, V. R. R.; Chmelik, C.; Weitkamp, J.; Kärger, J. J. Chem. Phys. 2011, 135, 184201.

(30) Ruthven, D. M.; Farooq, S.; Knaebel, K. S. Pressure Swing Adsorption; VCH Publishers: New York, 1994.

(31) Thompho, S.; Chanajaree, R.; Remsungnen, T.; Hannongbua, S.; Bopp, P. A.; Fritzsche, S. J. Phys. Chem. A 2009, 113, 2004-2014.

(32) June, R. L.; Bell, A. T.; Theodorou, D. N. J. Phys. Chem. 1991, 95, 8866-8878.

(33) Beerdsen, E.; Smit, B.; Dubbeldam, D. Phys. Rev. Lett. 2004, 93, 248301.

(34) Dubbeldam, D.; Beerdsen, E.; Vlugt, T. J. H.; Smit, B. J. Chem. Phys. 2005, 112, 224712.

(35) Veziri, C. M.; Palomino, M.; Karanikolos, G. N.; Corma, A.; Kanellopoulos, N. K.; Tsapatsis, M. Chem. Mater. 2010, 22, 14921502.

(36) Caro, J.; Noack, M.; Kölsch, P.; Schäfer, R. Microporous Mesoporous Mater. 2000, 38, 3-24.

(37) Caro, J.; Noack, M. Microporous Mesoporous Mater. 2008, 115, 215-233.

(38) Chandler, D. J. Chem. Phys. 1978, 68, 2959-2970.

(39) Frenkel, D.; Smit, B. Understanding Molecular Simulations: From Algorithms to Applications; Academic Press: San Diego, 2002.

(40) Schüring, A. J. Phys. Chem. C 2007, 111, 11285-11290.

(41) Pan, L.; Parker, B.; Huang, X. Y.; Olson, D. H.; Lee, J.; Li, J. J. Am. Chem. Soc. 2006, 128, 4180-4181.

(42) Zimmermann, N. E. R; Keil, F. J. Diffusion Fundamentals 2011, $16,1-2$. 